\title{
The Behavior of Domain Decomposition Methods When the Overlapping Length is Large.
}

\author{
Minh-Binh TRAN \\ Basque Center for Applied Mathematics \\ Mazarredo 14, \\ 48009 Bilbao Spain \\ Email: tbinh@bcamath.org
}

October 9, 2013

\begin{abstract}
In this paper, we introduce a new approach for the convergence problem of optimized Schwarz methods by studying a generalization of these methods for a semilinear elliptic equation. We study the behavior of the algorithm when the overlapping length is large.
\end{abstract}

Keyword Domain decomposition, Schwarz methods, semilinear elliptic equations.

MSC: $65 \mathrm{M} 12$.

\section{Introduction}

The Schwarz method was invented by Schwarz and then recovered by Lions in the three papers [12], [13], [14]. Based on the work of Lions in [12], the optimized Schwarz methods were introduced (see [7]) [8]), in order to improve the performance of classical Schwarz method, by using some new transmission conditions in the place of the Dichrichlet one, inspired by the work of Lions. However, there are not many tools to study the convergence 
of the methods. For the optimized Schwarz methods, the main techniques are to use the Fourier transform (see [7], [5], [8], [6], [2], [9]) and the energy estimates (see [10], [3], [11], [15]).

It is known through practical implementation of the algorithm that when the overlapping length is large, the algorithms always converge, but there is no rigorous proof for this. In this paper, we try to give a new approach to the convergence problem of optimized Schwarz methods, by studying theoretically the convergence problem when the overlapping length is large. We introduce a generalization of the optimized Schwarz methods for a semilinear elliptic equation and then study the wellposedness and convergence of the algorithm.

The paper is organized as follows. In Section 3, we introduce the definition of the algorithm, the main results and some notations that will be used in the next sections. The proof of Theorem 2.1, about the existence and uniqueness of the global problem, is given in Section 2. In Section 4, we prove the well-posedness of the algorithm. Section 5 is devoted to the proof of the convergence of the algorithm.

\section{The Main Results}

We consider the following class of elliptic equations

$$
-\Delta u(x)+c(x) u(x)=f(x, u(x)) \text { in } \Omega=(-R, R)^{N} \text {, and } u=0 \text { on } \partial \Omega \text {, }
$$

where $R$ is some large positive number, and impose the following conditions on it

(E1) $\forall \alpha, \beta$ in $\mathbb{R}$, for a.e. $x$ in $\Omega,|f(x, \alpha)-f(x, \beta)| \leq C|\alpha-\beta|$, where $C$ is a positive constant.

(E2) $c \in L^{\infty}(\Omega)$ and there exists $\lambda>0$ such that $0<C<\lambda \leq c(x)$ for a.e. $x$ in $\Omega$.

(E3) $f(., 0) \in L^{2}(\Omega)$.

Remark 2.1 Condition (E2) is assumpted in order to keep the coercivity of the elliptic operator as shown in the existence proof, which relies on the fixed point theorem for contraction maps. Indeed, the conditions that we present here are quite normal and generalizing the result to higher nonlinearity is a difficult task, and it goes beyond the purpose of our paper. Notice that similar types of elliptic operators were considered in [13] with Dirichlet boundary conditions. 
Theorem 2.1 Equation (2.1) has a unique solution in $H^{2}(\Omega)$.

We define a nonlinear optimized Schwarz (NOSM), which is a generalization of the optimized Schwarz methods (OSMs) with Robin transmission conditions, for Equation (2.1). Schwarz algorithm with these nonlinear Robin transmission condition in the nonoverlapping case where already proposed in [3]. However they have not been completely analyzed in that paper.

Definition 2.1 Divide $\Omega$ into two $\Omega_{1}, \Omega_{2}$ such that $\Omega_{1} \cup \Omega_{2}=\Omega$ and denote by $L$ the distance between $\partial \Omega_{1}$ and $\partial \Omega_{2}$. Start with the initial guesses $u_{1}^{0}$ in $H^{2}\left(\Omega_{1}\right)$ and $u_{2}^{0}$ in $H^{2}\left(\Omega_{2}\right)$, define the solutions at step $n$

$$
\begin{aligned}
& \left\{\begin{array}{l}
-\Delta u_{1}^{n}(x)+c(x) u_{1}^{n}(x)=f\left(x, u_{1}^{n}(x)\right) \text { in } \Omega_{1}, \\
\frac{\partial u_{1}^{n}}{\partial n_{1}}+p_{1}^{n}\left(u_{1}^{n}\right)=\frac{\partial u_{2}^{n-1}}{\partial n_{1}}+p_{1}^{n}\left(u_{2}^{n-1}\right) \text { on } \partial \Omega_{1},
\end{array}\right. \\
& \left\{\begin{array}{l}
-\Delta u_{2}^{n}(x)+c(x) u_{2}^{n}(x)=f\left(x, u_{2}^{n}(x)\right) \text { in } \Omega_{2}, \\
\frac{\partial u_{2}^{n}}{\partial n_{2}}+p_{2}^{n}\left(u_{2}^{n}\right)=\frac{\partial u_{1}^{n-1}}{\partial n_{2}}+p_{2}^{n}\left(u_{1}^{n-1}\right) \text { on } \partial \Omega_{2},
\end{array}\right.
\end{aligned}
$$

where $p_{1}^{n}, p_{2}^{n}$ are coefficients of the algorithm satisfying the following conditions.

(P1) $p_{1}^{n}$ and $p_{2}^{n}$ are continuous and increasing functions from $\mathbb{R}$ to $\mathbb{R} ; p_{1}^{n}(0)=$ $p_{2}^{n}(0)=0$.

(P2)There exists a positive constant $P$ such that, for all $n, \forall \zeta_{1}, \zeta_{2} \in \mathbb{R}$ such that $\left|p_{1}^{n}\left(\zeta_{1}\right)-p_{1}^{n}\left(\zeta_{2}\right)\right| \leq P\left|\zeta_{1}-\zeta_{2}\right|$ and $\left|p_{2}^{n}\left(\zeta_{1}\right)-p_{2}^{n}\left(\zeta_{2}\right)\right| \leq P\left|\zeta_{1}-\zeta_{2}\right|$.

Remark 2.2 If $p_{1}(\zeta)=p \zeta$ and $p_{2}(\zeta)=-q \zeta, \forall \zeta \in \mathbb{R}$ where $p$, $q$ are positive numbers, Algorithm (2.2) becomes the optimized domain decomposition methods with Robin transmission conditions in [2], [6], [7], [8], [5], [10]. With the purpose of giving an abstract theory of the convergence of the algorithm, we give a transmission condition which is more general than the usual ones.

Theorem 2.2 The NOSDDM defined in Definition 2.1 is well posed, in the sense that $(2.2)$ has solutions in $H^{2}\left(\Omega_{1}\right)$ and $H^{2}\left(\Omega_{2}\right)$ at each step.

Theorem 2.3 Denote the unique solution of (2.1) by $u$ and denote the errors at step $n$ to be $e_{1}^{n}, e_{2}^{n}$ (i.e. $e_{1}^{n}=u_{1}^{n}-u$ and $e_{2}^{n}=u_{2}^{n}-u$ ). There exists $L_{0}>0$ such that for $L>L_{0},\left\|e_{1}^{n}\right\|_{H^{1}\left(\Omega_{1}\right)}$ and $\left\|e_{2}^{n}\right\|_{H^{1}\left(\Omega_{2}\right)}$ tend to 0 as $n$ tends to infinity.

Remark 2.3 Finding the smallest value for $L_{0}$ to converge is an interesting problem that will be treated in a different paper. 


\section{Proof of Theorem 2.1}

Fix $v$ in $L^{2}(\Omega)$, by a standard Lax-Milgram argument, there exists a unique solution $w$ in $H^{1}(\Omega)$ of

$$
\int_{\Omega} \nabla w \nabla \phi d x+\int_{\Omega} c w \phi d x=\int_{\Omega} f(x, v) \phi d x, \forall \phi \in H^{1}(\Omega) .
$$

Define

$$
\begin{gathered}
T: L^{2}(\Omega) \rightarrow H^{1}(\Omega) \\
T v:=w .
\end{gathered}
$$

Let $v_{1}, v_{2}$ be in $L^{2}(\Omega)$. According to the definition of $T$

$$
\int_{\Omega} \nabla\left(T v_{1}\right) \nabla \phi d x+\int_{\Omega} c T v_{1} \phi d x=\int_{\Omega} f\left(x, v_{1}\right) \phi d x, \forall \phi \in H^{1}(\Omega),
$$

and

$$
\int_{\Omega} \nabla\left(T v_{1}\right) \nabla \phi d x+\int_{\Omega} c T v_{2} \phi d x=\int_{\Omega} f\left(x, v_{2}\right) \phi d x, \forall \phi \in H^{1}(\Omega) .
$$

subtract the two equations and choose $\phi$ to be $T v_{2}-T v_{1}$

$$
\int_{\Omega}\left(\left|\nabla\left(T v_{2}-T v_{1}\right)\right|^{2}+c\left|T v_{2}-T v_{1}\right|^{2}\right) d x=\int_{\Omega}\left[f\left(x, v_{2}\right)-f\left(x, v_{1}\right)\right]\left(T v_{2}-T v_{1}\right) d x
$$

Condition (E1) leads to

$$
\begin{aligned}
& \int_{\Omega}\left[f\left(x, v_{2}\right)-f\left(x, v_{1}\right)\right]\left(T v_{2}-T v_{1}\right) d x \\
\leq & C \int_{\Omega}\left|v_{2}-v_{1}\right|\left|T v_{2}-T v_{1}\right| d x \\
\leq & \frac{C}{2} \int_{\Omega}\left|v_{2}-v_{1}\right|^{2} d x+\frac{C}{2} \int_{\Omega}\left|T v_{2}-T v_{1}\right|^{2} d x .
\end{aligned}
$$

Equations (3.2) and (3.3) imply that

$$
\int_{\Omega}\left[\left|\nabla\left(T v_{2}-T v_{1}\right)\right|^{2}+\left(c-\frac{C}{2}\right)\left|T v_{2}-T v_{1}\right|^{2}\right] d x \leq \frac{C}{2} \int_{\Omega}\left|v_{2}-v_{1}\right|^{2} d x .
$$


Condition (E2) and Inequality (3.4) imply that

$$
\left\|T v_{2}-T v_{1}\right\|_{L^{2}(\Omega)} \leq \sqrt{\frac{C}{2 \lambda-C}}\left\|v_{2}-v_{1}\right\|_{L^{2}(\Omega)} .
$$

Since $\sqrt{\frac{C}{2 \lambda-C}}<1, T$ is a contraction from $L^{2}(\Omega)$ to $L^{2}(\Omega)$. Then there exists a unique $u$ in $L^{2}(\Omega)$ such that $T u=u$ and this is also the unique solution of

$$
\int_{\Omega} \nabla u \nabla \phi d x+\int_{\Omega} c u \phi d x=\int_{\Omega} f(x, u) \phi d x, \forall \phi \in H^{1}(\Omega) .
$$

The fact that $u$ belongs to $H^{2}(\Omega)$ can be inferred from the results in section $6.3,[4]$.

\section{$4 \quad$ Proof of Theorem 2.2}

The well-posedness of the algorithm, i.e. the existence and uniqueness of solutions to the subdomain problems follow from the theory of $(S)_{+}$operator in [17, Chapter 27$]$ ): we prove that the subequation is indeed an equation of an $(S)_{+}$operator and the existence result follows directly from the classical theory. Before proving the theorem, we recall the following definition (see [17, Chapter 27 ])

Definition 4.1 Let $h$ be a mapping from $H^{1}(\Omega)$ into $\left(H^{1}(\Omega)\right)^{*}$, we say that (i) $h$ is demicontinuous if it has the following property: Let $\left\{x_{n}\right\}$ be a sequence in $H^{1}(\Omega)$ such that $\left\{x_{n}\right\}$ converges strongly to $x$ in $H^{1}(\Omega)$, then $h\left(x_{n}\right)$ converges weakly to $h(x)$ in $H^{1}(\Omega)$.

(ii) $h$ is of class $(S)_{+}$if $h$ is demicontinuous and has the following property: Let $\left\{x_{n}\right\}$ be a sequence in $H^{1}(\Omega)$ such that $\left\{x_{n}\right\}$ converges weakly to $x$ in $H^{1}(\Omega)$. Then $\left\{x_{n}\right\}$ converges strongly to $x$ in $H^{1}(\Omega)$ provided $\limsup _{n \rightarrow \infty}$ $\left\langle h\left(x_{n}\right), x_{n}-x\right\rangle \leq 0$, where $\langle.,$.$\rangle is product in H^{1}$.

(iii) $h$ is pseudomonotone, if for any sequence $\left\{x_{n}\right\}$ such that $x_{n}$ converges weakly to $x$ in $H^{1}(\Omega)$ and $\limsup _{n \rightarrow \infty}\left\langle h\left(x_{n}\right), x_{n}-x\right\rangle \leq 0$, it follows that $\left\{h\left(x_{n}\right)\right\}$ converges weakly to $h(x)$ in $\left(H^{1}(\Omega)\right)^{*}$ and $\lim _{n \rightarrow \infty}\left\langle h\left(x_{n}\right), x_{n}-x\right\rangle=$ 0 .

Consider the following linear operators from $H^{1}$ to $H^{1}$

$$
\left\langle S_{1} w, \phi\right\rangle=\int_{\Omega} \nabla w \nabla \phi d x
$$




$$
\left\langle S_{2} w, \phi\right\rangle=\int_{\Omega}(c w-F(., w)) \phi d x-\int_{\partial \Omega}[g-p(w)] \phi d \omega,
$$

where $c, F$, satisfy Conditions $\left(E_{1}\right),\left(E_{2}\right)$, and $\left(E_{3}\right) ; p$ satisfies Conditions $\left(P_{1}\right),\left(P_{2}\right)$ of $p_{1}^{n}$ and $p_{2}^{n}$; and $g \in L^{2}(\partial \Omega)$.

Define

$$
\langle S w, \phi\rangle=\left\langle S_{1} w, \phi\right\rangle+\left\langle S_{2} w, \phi\right\rangle \text {. }
$$

Lemma 4.1 The operator $S$ defined in (4.2) is pseudomonotone.

Proof We prove the lemma in two steps.

Step 1: $S_{1}$ is an $(S)_{+}$and pseudomonotone operator on $H^{1}(\Omega)$.

Let $\left\{w_{n}\right\}$ be a sequence weakly converging to $w$ in $H^{1}(\Omega)$ such that $\lim \sup _{n \rightarrow \infty}\left\langle S_{1}\left(w_{n}\right), w_{n}-w\right\rangle \leq 0$. From the weak convergence property of $\left\{w_{n}\right\}$

$$
\lim _{n \rightarrow \infty} \int_{\Omega} \nabla w \nabla\left(w_{n}-w\right) d x=0
$$

We then infer

$$
\limsup _{n \rightarrow \infty} \int_{\Omega}\left|\nabla\left(w_{n}-w\right)\right|^{2} d x=0
$$

which leads to

$$
\lim _{n \rightarrow \infty} \int_{\Omega}\left|\nabla\left(w_{n}-w\right)\right|^{2} d x=0
$$

[1, Theorem 5.4] deduces that $\left\{w_{n}\right\}$ converges strongly to $w$ in $L^{2}(\Omega)$. Combining all the above facts, we get $w_{n}$ tends to $w$ as $n$ tends to infinity, which means $S_{1}$ is an $(S)_{+}$and then a pseudomonotone operator.

Step 2: $S$ is pseudomonotone.

Let $\left\{w_{n}\right\}$ be a sequence converging weakly to $w$ in $H^{1}(\Omega)$ such that $\lim \sup _{n \rightarrow \infty}\left\langle S\left(w_{n}\right), w_{n}-w\right\rangle \leq 0$. Note that

$$
\begin{aligned}
\left|\left\langle S_{2} w_{n}, w_{n}-w\right\rangle\right| \leq & \int_{\Omega}\left|c w_{n}-F\left(., w_{n}\right)\right|\left|w_{n}-w\right| d x+ \\
& +\int_{\partial \Omega}\left|g-p\left(w_{n}\right)\right|\left|w_{n}-w\right| d w
\end{aligned}
$$




$$
\begin{aligned}
\leq & {\left[\left(\|c\|_{L^{\infty}(\Omega)}+C\right)\left\|w_{n}\right\|_{L^{2}(\Omega)}+\|F(., 0)\|_{L^{2}(\Omega)}\right] \times } \\
& \times\left\|w_{n}-w\right\|_{L^{2}(\Omega)}+ \\
& +\left[\|g\|_{L^{2}(\partial \Omega)}+P\left\|w_{n}\right\|_{L^{2}(\partial \Omega)}+\|p(0)\|_{L^{2}(\partial \Omega)}\right] \times \\
& \times\left\|w_{n}-w\right\|_{L^{2}(\partial \Omega)} .
\end{aligned}
$$

Since $\left\{w_{n}\right\}$ is bounded in $H^{1}(\Omega)$, it is bounded in $L^{2}(\Omega)$, and their traces are bounded in $L^{2}(\partial \Omega)$. This implies the existence of a positive constant $K_{3}$ such that

$$
\left|\left\langle S_{2} w_{n}, w_{n}-w\right\rangle\right| \leq K_{3}\left[\left\|w_{n}-w\right\|_{L^{2}(\Omega)}+\left\|w_{n}-w\right\|_{L^{2}(\partial \Omega)}\right] .
$$

By the boundedness of $\left\{w_{n}\right\}$ in $H^{1}(\Omega)$,

$$
\begin{aligned}
& \lim _{n \rightarrow \infty}\left\|w_{n}-w\right\|_{L^{2}(\partial \Omega)}=0, \\
& \lim _{n \rightarrow \infty}\left\|w_{n}-w\right\|_{L^{2}(\Omega)}=0,
\end{aligned}
$$

which leads to

$$
\lim _{n \rightarrow \infty}\left|\left\langle S_{2} w_{n}, w_{n}-w\right\rangle\right|=0 .
$$

Combining with the fact that

$$
\limsup _{n \rightarrow \infty}\left\langle S w_{n}, w_{n}-w\right\rangle \leq 0
$$

we get

$$
\limsup _{n \rightarrow \infty}\left\langle S_{1} w_{n}, w_{n}-w\right\rangle \leq 0,
$$

which means $\left\{w_{n}\right\}$ converges strongly to $w$ in $H^{1}(\Omega)$, and as a consequence $S$ is pseudomonotone.

\section{Lemma $4.2 S$ is coercive.}

Proof In order to study the coercivity of $S$, we consider the quantity $\langle S w, w\rangle$ where $w$ is any element of $H^{1}(\Omega)$.

$$
\begin{aligned}
\langle S w, w\rangle & =\int_{\Omega}|\nabla w|^{2} d x+\int_{\Omega}[c w-F(., w)] w d x-\int_{\partial \Omega}[g-p(w)] w d \omega \\
& \geq \int_{\Omega}|\nabla w|^{2} d x+\int_{\Omega}(\lambda-C)|w|^{2} d x-\int_{\Omega} F(., 0) w d x+
\end{aligned}
$$




$$
\begin{aligned}
& +\int_{\partial \Omega}[-g+p(0)] w d \omega+\int_{\partial \Omega}[-p(0)+p(w)] w d \omega \\
\geq & \|\nabla w\|_{L^{2}(\Omega)}^{2}+(\lambda-C)\|w\|_{L^{2}(\Omega)}^{2}-\|F(., 0)\|\left\|_{L^{2}(\Omega)}\right\| w \|_{L^{2}(\Omega)}- \\
& -\left[\|g\|_{L^{2}(\partial \Omega)}+\|p(0)\| \|_{L^{2}(\partial \Omega)}\right]\|w\|_{L^{2}(\partial \Omega)},
\end{aligned}
$$

here we use the hypotheses (E1) and (P1).

From these calculations, we can see that

$$
\lim _{\|w\|_{H^{1}(\Omega)} \rightarrow \infty} \frac{\langle S w, w\rangle}{\|w\|_{H^{1}(\Omega)}}=+\infty .
$$

The coercivity of $S$ has been proved.

\section{Proof of Theorem 2.2 :}

We prove the well-posedness of the algorithm by induction. Suppose hat the algorithm is well posed up to step $k$, consider the problems on the subdomains at step $k+1$.

$$
\begin{aligned}
& \left\{\begin{array}{l}
-\Delta u_{1}^{k+1}(x)+c(x) u_{1}^{k+1}(x)=F\left(x, u_{1}^{k+1}(x)\right) \text { in } \Omega_{1}, \\
\frac{\partial u_{1}^{k+1}}{\partial n_{1}}+p_{1}^{k+1}\left(u_{1}^{k+1}\right)=\frac{\partial u_{2}^{k}}{\partial n_{1}}+p_{1}^{k+1}\left(u_{2}^{k}\right) \text { on } \partial \Omega_{1},
\end{array}\right. \\
& \left\{\begin{array}{l}
-\Delta u_{2}^{k+1}(x)+c(x) u_{2}^{k+1}(x)=F\left(x, u_{2}^{k+1}(x)\right) \text { in } \Omega_{2}, \\
\frac{\partial u_{2}^{k+1}}{\partial n_{2}}+p_{2}^{k+1}\left(u_{2}^{k+1}\right)=\frac{\partial u_{1}^{k}}{\partial n_{2}}+p_{2}^{k+1}\left(u_{1}^{k}\right) \text { on } \partial \Omega_{2},
\end{array}\right.
\end{aligned}
$$

Consider the problem on $\Omega_{1}$, since $u_{2}^{k}$ is in $H^{2}\left(\Omega_{2}\right), \frac{\partial u_{2}^{k}}{\partial n_{1}}+p_{1}^{k+1}\left(u_{2}^{k}\right)$ belongs to $L^{2}\left(\partial \Omega_{1}\right)$. As a result, the following operator is well defined

$$
\begin{aligned}
<S_{1}^{k+1} w, \phi>= & \int_{\Omega} \nabla w \nabla \phi d x+\int_{\Omega}(c w-F(., w)) \phi d x \\
& -\int_{\partial \Omega}\left[\frac{\partial u_{2}^{k}}{\partial n_{1}}+p_{1}^{k+1}\left(u_{2}^{k+1}\right)-p_{1}^{k+1}\left(u_{2}^{k}\right)\right] \phi d \omega,
\end{aligned}
$$

where $w, \phi \in H^{1}\left(\Omega_{1}\right)$. According to Lemma 4.1 and Lemma $4.2, S_{1}^{k+1}$ is pseudomonotone and coercive. Since $S_{1}^{k+1}$ is bounded, by [17, Theorem 27.A ], there exists a solution $w$ for the equation $\left\langle S_{1}^{k+1} w, \phi\right\rangle=0$. By the same technique as in [16, Theorem 3.25 ], $w$ belongs to $H^{2}\left(\Omega_{1}\right)$. Then the solution $u_{1}^{k+1}$ of the problem on $\Omega_{1}$ exists and belongs to $H^{2}\left(\Omega_{1}\right)$. Similarly the solution $u_{2}^{k+1}$ of the problem on $\Omega_{2}$ exists and belongs to $H^{2}\left(\Omega_{2}\right)$. Hence, the algorithm is well posed by induction. 


\section{$5 \quad$ Proof of Theorem 2.3}

We define some important notations which will be used later in the section.

- Define the function $\delta_{1}$

$$
\begin{aligned}
\delta_{1} & : \quad \Omega \rightarrow \mathbb{R}_{+} \\
x & \longmapsto \delta_{1}(x):=d\left(x, \partial \Omega_{1}\right)=\min _{y \in \partial \Omega_{1}} d(x, y),
\end{aligned}
$$

where $d$ is the Euclidean distance in $\Omega$.

- Define the function $\delta_{2}$

$$
\begin{aligned}
\delta_{2}: & \Omega \rightarrow \mathbb{R}_{+} \\
x & \longmapsto \delta_{2}(x):=d\left(x, \partial \Omega_{2}\right)=\min _{y \in \partial \Omega_{2}} d(x, y),
\end{aligned}
$$

where $d$ is the Euclidean distance in $\Omega$.

Let $\alpha, \beta$ be positive constants to be chosen later, for $x$ in $\Omega_{1}$, define

$$
D_{1}(x)=\left\{\begin{array}{l}
\alpha \delta_{1}(x)+\beta L \text { if } 0 \leq \delta_{1}(x) \leq L, \\
(\alpha+\beta) L \text { if } \delta_{1}(x)>L
\end{array}\right.
$$

and

$$
\Phi_{1}(x)=\exp \left(D_{1}(x)\right)
$$

then $\Phi_{1}$ belongs to $L^{\infty}\left(\Omega_{1}\right)$. Since $\delta_{1}$ is Lipschitz, $\delta_{1}$ is differentiable a.e. and the derivative belongs to $L^{\infty}\left(\Omega_{1}\right)$. The same is true for $\Phi_{1}$, which means

$$
\nabla \Phi_{1}(x)=\left\{\begin{array}{l}
\alpha \nabla \delta_{1}(x) \exp \left(D_{1}(x)\right) \text { if } 0 \leq \delta_{1}(x) \leq L, \\
0 \text { if } \delta_{1}(x)>L,
\end{array}\right.
$$

and $\nabla \Phi_{1}$ belongs to $L^{\infty}\left(\Omega_{1}\right)$.

Similarly, define for $x$ in $\Omega_{2}$,

$$
D_{2}(x)=\left\{\begin{array}{l}
\alpha \delta_{2}(x)+\beta L \text { if } 0 \leq \delta_{2}(x) \leq L, \\
(\alpha+\beta) L \text { if } \delta_{2}(x)>L
\end{array}\right.
$$


and

$$
\Phi_{2}(x)=\exp \left(D_{2}(x)\right)
$$

then $\Phi_{2}$ belongs to $L^{\infty}\left(\Omega_{2}\right)$ and

$$
\nabla \Phi_{2}(x)=\left\{\begin{array}{l}
\alpha \nabla \delta_{2}(x) \exp \left(D_{2}(x)\right) \text { if } 0 \leq \delta_{2}(x) \leq L, \\
0 \text { if } \delta_{2}(x)>L
\end{array}\right.
$$

and $\nabla \Phi_{2}$ belongs to $L^{\infty}\left(\Omega_{2}\right)$.

We consider the equation of $e_{1}^{n}$ on $\Omega_{1}$

$$
\left\{\begin{array}{l}
-\Delta e_{1}^{n}(x)+c(x) e_{1}^{n}(x)=f\left(x, u_{1}^{n}(x)\right)-f(x, u(x)) \text { in } \Omega_{1}, \\
\frac{\partial e_{1}^{n}}{\partial n_{1}}+\left[p_{1}^{n}\left(u_{1}^{n}\right)-p_{1}^{n}(u)\right]=\frac{\partial e_{2}^{n-1}}{\partial n_{1}}+\left[p_{1}^{n}\left(u_{2}^{n-1}\right)-p_{1}^{n}(u)\right] \text { on } \partial \Omega_{1} .
\end{array}\right.
$$

Put $\varphi=e_{1}^{n} \Phi_{1}$, then $\varphi$ belongs to $L^{2}\left(\Omega_{1}\right)$ as $\Phi_{1}$ belongs to $L^{\infty}\left(\Omega_{1}\right)$ and $e_{1}^{n}$ belongs to $L^{2}\left(\Omega_{1}\right)$. Moreover,

$$
\nabla \varphi=\nabla e_{1}^{n} \Phi_{1}+\nabla \Phi_{1} e_{1}^{n}
$$

which means $\nabla \varphi$ belongs to $L^{2}\left(\Omega_{1}\right)$. It follows that $\varphi$ belongs to $H^{1}\left(\Omega_{1}\right)$.

Use $\varphi$ as a test function for (5.1)

$$
\begin{aligned}
\int_{\Omega_{1}} \nabla e_{1}^{n} \nabla \varphi d x+\int_{\Omega_{1}} c e_{1}^{n} \varphi d x= & \int_{\Omega_{1}}\left[f\left(x, u_{1}^{n}\right)-f(x, u)\right] \varphi d x \\
& +\int_{\partial \Omega_{1}}\left[\frac{\partial e_{2}^{n-1}}{\partial n_{1}}+\left[p_{1}^{n}\left(u_{2}^{n-1}\right)-p_{1}^{n}(u)\right]\right] \varphi d \omega \\
& -\int_{\partial \Omega_{1}}\left[p_{1}^{n}\left(u_{1}^{n}\right)-p_{1}^{n}(u)\right] \varphi d \omega
\end{aligned}
$$

or

$$
\begin{aligned}
& \int_{\Omega_{1}} \nabla e_{1}^{n} \nabla\left(e_{1}^{n} \exp \left(D_{1}\right)\right) d x+\int_{\Omega_{1}} c\left(e_{1}^{n}\right)^{2} \exp \left(D_{1}\right) d x \\
= & \int_{\Omega_{1}}\left[f\left(x, u_{1}^{n}\right)-f(x, u)\right] e_{1}^{n} \exp \left(D_{1}\right) d x \\
+ & \int_{\partial \Omega_{1}}\left[\frac{\partial e_{2}^{n-1}}{\partial n_{1}}+\left[p_{1}^{n}\left(u_{2}^{n-1}\right)-p_{1}^{n}(u)\right]-\left[p_{1}^{n}\left(u_{1}^{n}\right)-p_{1}^{n}(u)\right]\right] e_{1}^{n} \exp (\beta L) d \omega .
\end{aligned}
$$


Consider the first term on the left hand side of (5.3)

$$
\begin{aligned}
& \int_{\Omega_{1}} \nabla e_{1}^{n} \nabla\left(e_{1}^{n} \exp \left(D_{1}\right)\right) d x \\
= & \int_{\Omega_{1}}\left|\nabla e_{1}^{n}\right|^{2} \exp \left(D_{1}\right) d x+\alpha \int_{\Omega_{1}} \nabla e_{1}^{n} e_{1}^{n} \nabla \delta_{1}(x) \exp \left(D_{1}\right) d x \\
= & \frac{1}{2} \int_{\Omega_{1}}\left(\left|\nabla e_{1}^{n}\right|+\alpha e_{1}^{n} \nabla \delta_{1}\right)^{2} \exp \left(D_{1}\right) d x \\
& -\frac{1}{2} \int_{\Omega_{1}} \alpha^{2}\left|e_{1}^{n}\right|^{2}\left|\nabla \delta_{1}\right|^{2} \exp \left(D_{1}\right) d x+\frac{1}{2} \int_{\Omega_{1}}\left|\nabla e_{1}^{n}\right|^{2} \exp \left(D_{1}\right) d x \\
\geq & \frac{1}{2} \int_{\Omega_{1}}\left|\nabla e_{1}^{n}\right|^{2} \exp \left(D_{1}\right) d x-\int_{\Omega_{1}} \frac{1}{2} \alpha^{2}\left(e_{1}^{n}\right)^{2} \exp \left(D_{1}\right)\left|\nabla \delta_{1}\right|^{2} d x .
\end{aligned}
$$

Use (5.4) in (5.3) to get

$$
\begin{aligned}
& \int_{\Omega_{1}} \frac{1}{2}\left|\nabla e_{1}^{n}\right|^{2} \exp \left(D_{1}\right) d x+\int_{\Omega_{1}}\left[c-\frac{1}{2} \alpha^{2}\left|\nabla \delta_{1}\right|^{2}\right]\left(e_{1}^{n}\right)^{2} \exp \left(D_{1}\right) d x \\
\leq & \int_{\Omega_{1}}\left[f\left(x, u_{1}^{n}\right)-f(x, u)\right] e_{1}^{n} \exp \left(D_{1}\right) d x \\
& +\int_{\partial \Omega_{1}}\left[\frac{\partial e_{2}^{n-1}}{\partial n_{1}}+\left[p_{1}^{n}\left(u_{2}^{n-1}\right)-p_{1}^{n}(u)\right]-\left[p_{1}^{n}\left(u_{1}^{n}\right)-p_{1}^{n}(u)\right]\right] e_{1}^{n} \exp (\beta L) d \omega \\
\leq & \int_{\Omega_{1}}\left[f\left(x, u_{1}^{n}\right)-f(x, u)\right] e_{1}^{n} \exp \left(D_{1}\right) d x \\
& +\int_{\partial \Omega_{1}}\left[\frac{\partial e_{2}^{n-1}}{\partial n_{1}}+\left[p_{1}^{n}\left(u_{2}^{n-1}\right)-p_{1}^{n}(u)\right]\right] e_{1}^{n} \exp (\beta L) d \omega,
\end{aligned}
$$

here we use Condition (P1). Suppose that $\alpha<\sqrt{2 \lambda}$, consider the first term on the right hand side of (5.5)

$$
\begin{aligned}
& \int_{\Omega_{1}}\left[f\left(x, u_{1}^{n}\right)-f(x, u)\right] e_{1}^{n} \exp \left(D_{1}\right) d x \\
= & \int_{\Omega_{1}}\left[f\left(x, u_{1}^{n}\right)-f(x, u)\right] e_{1}^{n} \exp \left(D_{1}\right)\left[\lambda-\frac{1}{2} \alpha^{2}\left|\nabla \delta_{1}\right|^{2}\right]^{-\frac{1}{2}} \times \\
& \times\left[\lambda-\frac{1}{2} \alpha^{2}\left|\nabla \delta_{1}\right|^{2}\right]^{\frac{1}{2}} d x \\
\leq & \frac{1}{2} \int_{\Omega_{1}}\left[f\left(x, u_{1}^{n}\right)-f(x, u)\right]^{2} \exp \left(D_{1}\right)\left[\lambda-\frac{1}{2} \alpha^{2}\left|\nabla \delta_{1}\right|^{2}\right]^{-1} d x+
\end{aligned}
$$




$$
\begin{aligned}
& +\frac{1}{2} \int_{\Omega_{1}}\left(e_{1}^{n}\right)^{2} \exp \left(D_{1}\right)\left[\lambda-\frac{1}{2} \alpha^{2}\left|\nabla \delta_{1}\right|^{2}\right] d x \\
\leq & \frac{1}{2} \int_{\Omega_{1}} C^{2}\left(e_{1}^{n}\right)^{2} \exp \left(D_{1}\right)\left[\lambda-\frac{1}{2} \alpha^{2}\left|\nabla \delta_{1}\right|^{2}\right]^{-1} d x+ \\
& +\frac{1}{2} \int_{\Omega_{1}}\left(e_{1}^{n}\right)^{2} \exp \left(D_{1}\right)\left[\lambda-\frac{1}{2} \alpha^{2}\left|\nabla \delta_{1}\right|^{2}\right] d x .
\end{aligned}
$$

Using (5.6) in (5.5), we get

$$
\begin{aligned}
& \int_{\Omega_{1}} \frac{1}{2}\left|\nabla e_{1}^{n}\right|^{2} \exp \left(D_{1}\right) d x+ \\
& +\frac{1}{2} \int_{\Omega_{1}}\left[\left[\lambda-\frac{1}{2} \alpha^{2}\left|\nabla \delta_{1}\right|^{2}\right]-C^{2}\left[\lambda-\frac{1}{2} \alpha^{2}\left|\nabla \delta_{1}\right|^{2}\right]^{-1}\right] \times \\
& \times\left(e_{1}^{n}\right)^{2} \exp \left(D_{1}\right) d x \\
\leq & \int_{\partial \Omega_{1}}\left[\frac{\partial e_{2}^{n-1}}{\partial n_{1}}+\left[p_{1}^{n}\left(u_{2}^{n-1}\right)-p_{1}^{n}(u)\right]\right] e_{1}^{n} \exp (\beta L) d \omega .
\end{aligned}
$$

Consider the function $s(t)=t-C^{2} t^{-1}$ with $t$ in $[C,+\infty)$, we have $s^{\prime}(t)=$ $1+C^{2} t^{-2}>0$, then $s(t)$ is increasing. This implies

$$
\begin{aligned}
& {\left[\lambda-\frac{1}{2} \alpha^{2}\left|\nabla \delta_{1}\right|^{2}\right]-C^{2}\left[\lambda-\frac{1}{2} \alpha^{2}\left|\nabla \delta_{1}\right|^{2}\right]^{-1} } \\
\geq & \left(\lambda-\frac{1}{2} \alpha^{2}\right)-C^{2}\left(\lambda-\frac{1}{2} \alpha^{2}\right)^{-1} .
\end{aligned}
$$

Replace (5.8) into (5.7) to get

$$
\begin{aligned}
& \int_{\Omega_{1}} \frac{1}{2}\left|\nabla e_{1}^{n}\right|^{2} \exp \left(D_{1}\right) d x+\frac{1}{2} \int_{\Omega_{1}}\left[\left(\lambda-\frac{1}{2} \alpha^{2}\right)-C^{2}\left(\lambda-\frac{1}{2} \alpha^{2}\right)^{-1}\right]\left(e_{1}^{n}\right)^{2} \exp \left(D_{1}\right) d x \\
\leq & \int_{\partial \Omega_{1}}\left[\frac{\partial e_{2}^{n-1}}{\partial n_{1}}+\left[p_{1}^{n}\left(u_{2}^{n-1}\right)-p_{1}^{n}(u)\right]\right] e_{1}^{n} \exp (\beta L) d \omega .
\end{aligned}
$$

Let $r_{1}^{n}$ be an extension of $e_{1}^{n}$ in the following sense $r_{1}^{n}$ is in $H^{1}\left(\Omega \backslash \Omega^{o} \Omega_{1}\right)$, such that the traces of $r_{1}^{n}$ and $e_{1}^{n}$ are equal on $\partial \Omega_{1}$ and $\left\|r_{1}^{n}\right\|_{L^{2}\left(\Omega \backslash \Omega_{1}\right)} \leq C_{1}\left\|e_{1}^{n}\right\|_{L^{2}\left(\Omega_{1}\right)}$ and $\left\|r_{1}^{n}\right\|_{H^{1}\left(\Omega \backslash \Omega_{1}\right)}^{o} \leq C_{1}\left\|e_{1}^{n}\right\|_{H^{1}\left(\Omega_{1}\right)}$.

Replace $e_{1}^{n}$ by $r_{1}^{n}$ in the last term on the right hand side of (5.9),

$$
\int_{\Omega_{1}} \frac{1}{2}\left|\nabla e_{1}^{n}\right|^{2} \exp \left(D_{1}\right) d x+\frac{1}{2} \int_{\Omega_{1}}\left[\left(\lambda-\frac{1}{2} \alpha^{2}\right)-C^{2}\left(\lambda-\frac{1}{2} \alpha^{2}\right)^{-1}\right]\left(e_{1}^{n}\right)^{2} \exp \left(D_{1}\right) d x
$$




$$
\leq \int_{\partial \Omega_{1}}\left[\frac{\partial e_{2}^{n-1}}{\partial n_{1}}+\left[p_{1}^{n}\left(u_{2}^{n-1}\right)-p_{1}^{n}(u)\right]\right] r_{1}^{n} \exp (\beta L) d \omega
$$

which implies

$$
\begin{aligned}
\int_{\partial \Omega_{1}} \frac{\partial e_{2}^{n-1}}{\partial n_{1}} r_{1}^{n} \exp (\beta L) d \omega= & \int_{\Omega \backslash \Omega_{1}^{o}} \Delta e_{2}^{n-1} r_{1}^{n} \exp (\beta L) d x+ \\
& +\int_{\Omega \backslash \Omega_{1}} \nabla e_{2}^{n-1} \nabla r_{1}^{n} \exp (\beta L) d x \\
= & \int_{\Omega \backslash \Omega_{1}}\left[c e_{2}^{n-1}-\left[f\left(u_{2}^{n-1}\right)-f(u)\right]\right] r_{1}^{n} \exp (\beta L) d x+ \\
& +\int_{\Omega \backslash \Omega_{1}} \nabla e_{2}^{n-1} \nabla r_{1}^{n} \exp (\beta L) d x
\end{aligned}
$$

Replace (5.11) into (5.10) to get

$$
\begin{aligned}
& \left.\int_{\Omega_{1}}\left|\nabla e_{1}^{n}\right|^{2} \exp \left(D_{1}\right) d x+\int_{\Omega_{1}}\left[\left(\lambda-\frac{1}{2} \alpha^{2}\right)-C^{2}\left(\lambda-\frac{1}{2} \alpha^{2}\right)^{-1}\right]\left(e_{1}^{n}\right)^{2} \exp \left(D_{1}\right)\right) d x \\
\leq & 2 \int_{\partial \Omega_{1}}\left[p_{1}^{n}\left(u_{2}^{n-1}\right)-p_{1}^{n}(u)\right] r_{1}^{n} \exp (\beta L) d \omega+ \\
& +2 \int_{\Omega \backslash \Omega_{1}}\left[c e_{2}^{n-1}-\left[f\left(u_{2}^{n-1}\right)-f(u)\right]\right] r_{1}^{n} \exp (\beta L) d x+ \\
& +2 \int_{\Omega \backslash \Omega_{1}} \nabla e_{2}^{n-1} \nabla r_{1}^{n} \exp (\beta L) d x
\end{aligned}
$$

Consider the first term on the right hand side of (5.12), by the trace theorem

$$
\begin{aligned}
& 2 \int_{\partial \Omega_{1}}\left[p_{1}^{n}\left(u_{2}^{n-1}\right)-p_{1}^{n}(u)\right] r_{1}^{n} \exp (\beta L) d \omega \\
\leq & \frac{P_{1}}{\epsilon_{1}^{n}} \int_{\partial \Omega_{1}}\left|e_{2}^{n-1}\right|^{2} \exp (2 \beta L) d \omega+\epsilon_{1}^{n} P_{1} \int_{\partial \Omega_{1}}\left|r_{1}^{n}\right|^{2} d \omega \\
\leq & \left.\exp (2 \beta L) \frac{P_{1} M_{1}^{2}}{\epsilon_{1}^{n}} \int_{\Omega_{\backslash} \Omega_{1}}\left(\left|e_{2}^{n-1}\right|^{2}+\left|\nabla e_{2}^{n-1}\right|^{2}\right) d x+\epsilon_{1}^{n} M_{1}^{2} P_{1} \int_{\Omega_{1}}\left(\left|e_{1}^{n}\right|^{2}+\left|\nabla e_{1}^{n}\right|^{2} 5 d 1\right]\right)
\end{aligned}
$$

where $M_{1}$ is a constant and $\epsilon_{1}^{n}$ is a constant to be chosen later.

Consider the integral in the first term on the right hand side of (5.13), we have the following estimate

$$
\int_{\Omega \backslash \Omega_{1}}\left(\left|e_{2}^{n-1}\right|^{2}+\left|\nabla e_{2}^{n-1}\right|^{2}\right) d x \leq \int_{\Omega \backslash \Omega_{1}}\left(\left|e_{2}^{n-1}\right|^{2}+\left|\nabla e_{2}^{n-1}\right|^{2}\right) \exp \left(D_{2}\right) \exp \left(-D_{2}\right) d x
$$




$$
\left.\leq \exp (-(\alpha+\beta) L) \int_{\Omega_{2}}\left(\left|e_{2}^{n-1}\right|^{2}+\left|\nabla e_{2}^{n-1}\right|^{2}\right) \exp (\not(5)) d L 4\right)
$$

Replace (5.14) into (5.13)

$$
\begin{aligned}
& 2 \int_{\partial \Omega_{1}}\left[p_{1}^{n}\left(u_{2}^{n-1}\right)-p_{1}^{n}(u)\right] r_{1}^{n} d \omega \\
\leq & \frac{P_{1} M_{1}^{2} \exp ((\beta-\alpha) L)}{\epsilon_{1}^{n}} \int_{\Omega_{2}}\left(\left|e_{2}^{n-1}\right|^{2}+\left|\nabla e_{2}^{n-1}\right|^{2}\right) \exp \left(D_{2}\right) d x+ \\
& +\epsilon_{1}^{n} M_{1}^{2} P_{1} \int_{\Omega_{1}}\left(\left|e_{1}^{n}\right|^{2}+\left|\nabla e_{1}^{n}\right|^{2}\right) \exp \left(D_{1}\right) d x .
\end{aligned}
$$

Using the same argument for the second term on the right hand side of (5.12), we have the estimate

$$
\begin{aligned}
& 2 \int_{\Omega \backslash \Omega_{1}}\left[c e_{2}^{n-1}-\left[f\left(u_{2}^{n-1}\right)-f(u)\right]\right] r_{1}^{n} \exp (\beta L) d x \\
\leq & 2\left(\|c\|_{\infty}+C\right) \int_{\Omega \backslash \Omega_{1}}\left|e_{2}^{n-1} \| r_{1}^{n}\right| \exp (\beta L) d x \\
\leq & \frac{\|c\|_{\infty}+C}{\epsilon_{1}^{n}} \exp ((\beta-\alpha) L) \int_{\Omega_{2}}\left|e_{2}^{n-1}\right|^{2} \exp \left(D_{2}\right) d x+ \\
& +\epsilon_{1}^{n}\left(\|c\|_{\infty}+C\right) C_{1}^{2} \int_{\Omega_{1}}\left|e_{1}^{n}\right|^{2} \exp \left(D_{1}\right) d x .
\end{aligned}
$$

Now, we estimate the last terms on the right hand side of (5.12)

$$
\begin{aligned}
& \int_{\Omega \backslash \Omega_{1}} 2 \nabla e_{2}^{n-1} \nabla r_{1}^{n} \exp (\beta L) d x \\
\leq & \frac{\exp ((\beta-\alpha) L)}{\epsilon_{1}^{n}} \int_{\Omega_{2}}\left|\nabla e_{2}^{n-1}\right|^{2} \exp \left(D_{2}\right) d x+ \\
& +\epsilon_{1}^{n} \int_{\Omega \backslash \Omega_{1}^{o}}\left|\nabla r_{1}^{n}\right|^{2} \exp (\beta L) d x \\
\leq & \frac{\exp ((\beta-\alpha) L)}{\epsilon_{1}^{n}} \int_{\Omega_{2}}\left|\nabla e_{2}^{n-1}\right|^{2} \exp \left(D_{2}\right) d x+ \\
& +\epsilon_{1}^{n} C_{1}^{2} \int_{\Omega_{1}}\left|\nabla e_{1}^{n}\right|^{2} \exp \left(D_{1}\right) d x+\epsilon_{1}^{n} C_{1}^{2} \int_{\Omega_{1}}\left|e_{1}^{n}\right|^{2} \exp \left(D_{1}\right) d x .
\end{aligned}
$$

Combine (5.15), (5.16), (5.17) with (5.12), we get

$$
\left.\int_{\Omega_{1}}\left|\nabla e_{1}^{n}\right|^{2} \exp \left(D_{1}\right) d x+\int_{\Omega_{1}}\left[\left(\lambda-\frac{\alpha^{2}}{2}\right)-C^{2}\left(\lambda-\frac{\alpha^{2}}{2}\right)^{-1}\right]\left(e_{1}^{n}\right)^{2} \exp \left(D_{1}\right)\right) d x
$$




$$
\begin{aligned}
\leq & \epsilon_{1}^{n}\left[P_{1} M_{1}^{2}+\left(\|c\|_{\infty}+C+1\right) C_{1}^{2}\right] \int_{\Omega_{1}}\left|e_{1}^{n}\right|^{2} \exp \left(D_{1}\right) d x+\left(\epsilon_{1}^{n} P_{1} M_{1}^{2}+C_{1}^{2} \epsilon_{1}^{n}\right) \int_{\Omega_{1}}\left|\nabla e_{1}^{n}\right|^{2} \exp \left(D_{1}\right) d x \\
& +\frac{\exp ((\beta-\alpha) L)}{\epsilon_{1}^{n}}\left(P_{1} M_{1}^{2}+\|c\|_{\infty}+C\right) \int_{\Omega_{2}}\left|e_{2}^{n-1}\right|^{2} \exp \left(D_{2}\right) d x+ \\
& +C \frac{\exp ((\beta-\alpha) L)}{\epsilon_{1}^{n}} \int_{\Omega_{2}}\left|\nabla e_{2}^{n-1}\right|^{2} \exp \left(D_{2}\right) d x .
\end{aligned}
$$

Which implies

$$
\begin{aligned}
& \left(1-\left(C_{1}^{2}+P_{1} M_{1}^{2}\right) \epsilon_{1}^{n}\right) \int_{\Omega_{1}}\left|\nabla e_{1}^{n}\right|^{2} \exp \left(D_{1}\right) d x+ \\
& +\int_{\Omega_{1}}\left[\left(\lambda-\frac{\alpha^{2}}{2}\right)-C^{2}\left(\lambda-\frac{\alpha^{2}}{2}\right)^{-1}-\epsilon_{1}^{n}\left[P_{1} M_{1}^{2}+\left(\|c\|_{\infty}+C+1\right) C_{1}^{2}\right]\right]\left(e_{1}^{n}\right)^{2} \exp \left(D_{1}\right) d x \\
\leq & \frac{\exp ((\beta-\alpha) L)}{\epsilon_{1}^{n}}\left(P_{1} M_{1}^{2}+\|c\|_{\infty}+C\right) \int_{\Omega_{2}}\left|e_{2}^{n-1}\right|^{2} \exp \left(D_{2}\right) d x+ \\
& +C \frac{\exp ((\beta-\alpha) L)}{\epsilon_{1}^{n}} \int_{\Omega_{2}}\left|\nabla e_{2}^{n-1}\right|^{2} \exp \left(D_{2}\right) d x
\end{aligned}
$$

Similarly, we have the following estimate for the errors on the subdomain $\Omega_{2}$

$$
\begin{aligned}
& \left(1-\left(C_{2}^{2}+P_{2} M_{2}^{2}\right) \epsilon_{2}^{n}\right) \int_{\Omega_{2}}\left|\nabla e_{2}^{n}\right|^{2} \exp \left(D_{2}\right) d x \\
& +\int_{\Omega_{2}}\left[\left(\lambda-\frac{\alpha^{2}}{2}\right)-C^{2}\left(\lambda-\frac{\alpha^{2}}{2}\right)^{-1}-\epsilon_{2}^{n}\left[P_{2} M_{2}^{2}+\left(\|c\|_{\infty}+C+1\right) C_{2}^{2}\right]\right]\left(e_{1}^{n}\right)^{2} \exp \left(D_{2}\right) d x \\
\leq & \frac{\exp ((\beta-\alpha) L)}{\epsilon_{2}^{n}}\left(P_{2} M_{2}^{2}+\|c\|_{\infty}+C\right) \int_{\Omega_{1}}\left|e_{1}^{n-1}\right|^{2} \exp \left(D_{1}\right) d x+ \\
& +C \frac{\exp ((\beta-\alpha) L)}{\epsilon_{2}^{n}} \int_{\Omega_{1}}\left|\nabla e_{1}^{n-1}\right|^{2} \exp \left(D_{1}\right) d x
\end{aligned}
$$

Choosing $\beta<\alpha<\sqrt{2} \lambda$, and put

$$
\begin{aligned}
A_{1}^{n} & =1-\left(C_{1}^{2}+P_{1} M_{1}^{2}\right)^{2} \epsilon_{1}^{n} \\
A_{2}^{n} & =1-\left(C_{2}^{2}+P_{2} M_{2}^{2}\right) \epsilon_{2}^{n} \\
A_{3}^{n} & =\left(\lambda-\frac{\alpha^{2}}{2}\right)-C^{2}\left(\lambda-\frac{\alpha^{2}}{2}\right)^{-1}-\epsilon_{1}^{n}\left[P_{1} M_{1}^{2}+\left(\|c\|_{\infty}+C+1\right) C_{1}^{2}\right], \\
A_{4}^{n} & =\left(\lambda-\frac{\alpha^{2}}{2}\right)-C^{2}\left(\lambda-\frac{\alpha^{2}}{2}\right)^{-1}-\epsilon_{2}^{n}\left[P_{2} M_{2}^{2}+\left(\|c\|_{\infty}+C+1\right) C_{2}^{2}\right], \\
B_{1}^{n} & =C \frac{\exp ((\beta-\alpha) L)}{\epsilon_{1}^{n}}
\end{aligned}
$$




$$
\begin{aligned}
& B_{2}^{n}=C \frac{\exp ((\beta-\alpha) L)}{\epsilon_{2}^{n}}, \\
& B_{3}^{n}=\left(P_{2} M_{2}^{2}+\|c\|_{\infty}+C\right) \frac{\exp ((\beta-\alpha) L}{\epsilon_{2}^{n}}, \\
& B_{4}^{n}=\left(P_{1} M_{1}^{2}+\|c\|_{\infty}+C\right) \frac{\exp ((\beta-\alpha) L)}{\epsilon_{2}^{n}},
\end{aligned}
$$

then sum (5.19) and (5.20) with the notations (5.21) from 2 to $m$ to get

$$
\begin{aligned}
& \sum_{n=2}^{m} \int_{\Omega_{1}} A_{1}^{n}\left|\nabla e_{1}^{n}\right|^{2} \exp \left(D_{1}\right) d x+\sum_{n=2}^{m} \int_{\Omega_{2}} A_{2}^{n}\left|\nabla e_{2}^{n}\right|^{2} \exp \left(D_{2}\right) d x+ \\
& +\sum_{n=2}^{m} \int_{\Omega_{1}} A_{3}^{n}\left|e_{1}^{n}\right|^{2} \exp \left(D_{1}\right) d x+\sum_{n=2}^{m} \int_{\Omega_{2}} A_{4}^{n}\left|e_{2}^{n}\right|^{2} \exp \left(D_{2}\right) d x \\
\leq & \sum_{n=1}^{m-1} \int_{\Omega_{1}} B_{1}^{n}\left|\nabla e_{1}^{n-1}\right|^{2} \exp \left(D_{1}\right) d x+\sum_{n=1}^{m-1} \int_{\Omega_{2}} B_{2}^{n}\left|\nabla e_{2}^{n-1}\right|^{2} \exp \left(D_{2}\right) d x \\
& +\sum_{n=1}^{m-1} \int_{\Omega_{1}} B_{3}^{n}\left|e_{1}^{n-1}\right|^{2} \exp \left(D_{1}\right) d x+\sum_{n=1}^{m-1} \int_{\Omega_{2}} B_{4}^{n}\left|e_{2}^{n-1}\right|^{2} \exp \left(D_{2}\right) d x,
\end{aligned}
$$

which implies

$$
\begin{aligned}
& \int_{\Omega_{1}} A_{1}^{m}\left|\nabla e_{1}^{m}\right|^{2} \exp \left(D_{1}\right) d x+\int_{\Omega_{2}} A_{2}^{m}\left|\nabla e_{2}^{m}\right|^{2} \exp \left(D_{2}\right) d x+ \\
& +\int_{\Omega_{1}} A_{3}^{m}\left|e_{1}^{m}\right|^{2} \exp \left(D_{1}\right) d x+\int_{\Omega_{2}} A_{4}^{m}\left|e_{2}^{m}\right|^{2} \exp \left(D_{2}\right) d x \\
& +\sum_{n=2}^{m-1} \int_{\Omega_{1}}\left(A_{1}^{n}-B_{1}^{n}\right)\left|\nabla e_{1}^{n}\right|^{2} \exp \left(2 D_{1}\right) d x+\sum_{n=2}^{m-1} \int_{\Omega_{2}}\left(A_{2}^{n}-B_{2}^{n}\right)\left|\nabla e_{2}^{n}\right|^{2} \exp \left(D_{2}\right) d x+ \\
& +\left(A_{3}^{n}-B_{3}^{n}\right) \sum_{n=2}^{m-1} \int_{\Omega_{1}}\left|e_{1}^{n}\right|^{2} \exp \left(2 D_{1}\right) d x+\left(A_{4}^{n}-B_{4}^{n}\right) \sum_{n=2}^{m-1} \int_{\Omega_{2}}\left|e_{2}^{n}\right|^{2} \exp \left(D_{2}\right) d x \\
\leq & \int_{\Omega_{1}} B_{1}^{1}\left|\nabla e_{1}^{1}\right|^{2} \exp \left(D_{1}\right) d x+\int_{\Omega_{2}} B_{2}^{1}\left|\nabla e_{2}^{1}\right|^{2} \exp \left(D_{2}\right) d x \\
& +\int_{\Omega_{1}} B_{3}^{1}\left|e_{1}^{1}\right|^{2} \exp \left(2 D_{1}\right) d x+\int_{\Omega_{2}} B_{4}^{1}\left|e_{2}^{1}\right|^{2} \exp \left(D_{2}\right) d x
\end{aligned}
$$

For $L_{0}$ large enough (since $R$ is large), by choosing suitable values of $\epsilon_{1}^{n}$ and $\epsilon_{2}^{n}$, we can infer that there exist $A_{1}, A_{2}, A_{3}, A_{4}, A_{5}, A_{6}, A_{7}, A_{8}>0$ such that

$$
\int_{\Omega_{1}} A_{1}\left|\nabla e_{1}^{m}\right|^{2} \exp \left(D_{1}\right) d x+\int_{\Omega_{2}} A_{2}\left|\nabla e_{2}^{m}\right|^{2} \exp \left(D_{2}\right) d x+
$$




$$
\begin{aligned}
& \quad+\int_{\Omega_{1}} A_{3}\left|e_{1}^{m}\right|^{2} \exp \left(D_{1}\right) d x+\int_{\Omega_{2}} A_{4}\left|e_{2}^{m}\right|^{2} \exp \left(D_{2}\right) d x \\
& \quad \sum_{n=2}^{m-1} \int_{\Omega_{1}} A_{5}\left|\nabla e_{1}^{n}\right|^{2} \exp \left(D_{1}\right) d x+\sum_{n=2}^{m-1} \int_{\Omega_{2}} A_{6}\left|\nabla e_{2}^{n}\right|^{2} \exp \left(D_{2}\right) d x+ \\
& +A_{7} \sum_{n=2}^{m-1} \int_{\Omega_{1}}\left|e_{1}^{n}\right|^{2} \exp \left(D_{1}\right) d x+A_{8} \sum_{n=2}^{m-1} \int_{\Omega_{2}}\left|e_{2}^{n}\right|^{2} \exp \left(D_{2}\right) d x \\
& \leq \int_{\Omega_{1}} B_{1}^{1}\left|\nabla e_{1}^{1}\right|^{2} \exp \left(D_{1}\right) d x+\int_{\Omega_{2}} B_{2}^{1}\left|\nabla e_{2}^{1}\right|^{2} \exp \left(D_{2}\right) d x \\
& +\int_{\Omega_{1}} B_{3}^{1}\left|e_{1}^{1}\right|^{2} \exp \left(D_{1}\right) d x+\int_{\Omega_{2}} B_{4}^{1}\left|e_{2}^{1}\right|^{2} \exp \left(D_{2}\right) d x,
\end{aligned}
$$

which means

$$
\begin{aligned}
& \lim _{n \rightarrow \infty} \int_{\Omega_{1}}\left|\nabla e_{1}^{n}\right|^{2} \exp \left(D_{1}\right) d x=0, \\
& \lim _{n \rightarrow \infty} \int_{\Omega_{2}}\left|\nabla e_{2}^{n}\right|^{2} \exp \left(D_{2}\right) d x=0, \\
& \lim _{n \rightarrow \infty} \int_{\Omega_{1}}\left|e_{1}^{n}\right|^{2} \exp \left(D_{1}\right) d x=0, \\
& \lim _{n \rightarrow \infty} \int_{\Omega_{2}}\left|e_{2}^{n}\right|^{2} \exp \left(D_{2}\right) d x=0
\end{aligned}
$$

As a consequence,

$$
\begin{aligned}
& \lim _{n \rightarrow \infty}\left\|u_{1}^{n}\right\|_{H^{1}\left(\Omega_{1}\right)}=0, \\
& \lim _{n \rightarrow \infty}\left\|u_{2}^{n}\right\|_{H^{1}\left(\Omega_{2}\right)}=0 .
\end{aligned}
$$

The theorem has been proved.

Acknowledgement. The author would like to thank the editor for his very kind invitation of writing this paper for the Central European Journal of Mathematics. He also wants to express his gratitude to the referees for their careful reading of the manuscript. The author has been supported by Grant MTM2011-29306-C02-00, MICINN, Spain, ERC Advanced Grant FP7-246775 NUMERIWAVES, and Grant PI2010-04 of the Basque Government. 


\section{References}

11] R. A. Adams. Sobolev Spaces. Academic Press [A subsidiary of Harcourt Brace Jovanovich, Publishers], New York-London, 1975. Pure and Applied Mathematics, Vol. 65.

[2] D. Bennequin, M. J. Gander, and L. Halpern. A homographic best approximation problem with application to optimized Schwarz waveform relaxation. Math. Comp., 78(265):185-223, 2009.

[3] Filipa Caetano, Martin J. Gander, Laurence Halpern, and Jérémie Szeftel. Schwarz waveform relaxation algorithms with nonlinear transmission conditions for reaction-diffusion equations. In Domain decomposition methods in science and engineering XIX, volume 78 of Lect. Notes Comput. Sci. Eng., pages 245-252. Springer, Heidelberg, 2011.

[4] L. C. Evans. Partial differential equations, volume 19 of Graduate Studies in Mathematics. American Mathematical Society, Providence, RI, 1998.

[5] M. J. Gander. A waveform relaxation algorithm with overlapping splitting for reaction diffusion equations. Numer. Linear Algebra Appl., 6(2):125-145, 1999. Czech-US Workshop in Iterative Methods and Parallel Computing, Part 2 (Milovy, 1997).

[6] M. J. Gander and L. Halpern. Optimized Schwarz waveform relaxation methods for advection reaction diffusion problems. SIAM J. Numer. Anal., 45(2):666-697 (electronic), 2007.

[7] M. J. Gander, L. Halpern, and F. Nataf. Optimal convergence for overlapping and non-overlapping Schwarz waveform relaxation. In Eleventh International Conference on Domain Decomposition Methods (London, 1998), pages 27-36 (electronic). DDM.org, Augsburg, 1999.

[8] M. J. Gander, L. Halpern, and F. Nataf. Optimized Schwarz methods. In Domain decomposition methods in sciences and engineering (Chiba, 1999), pages 15-27 (electronic). DDM.org, Augsburg, 2001.

[9] Martin J. Gander. Optimized Schwarz methods. SIAM J. Numer. Anal., 44(2):699-731 (electronic), 2006. 
[10] L. Halpern and J. Szeftel. Nonlinear nonoverlapping Schwarz waveform relaxation for semilinear wave propagation. Math. Comp., 78(266):865889, 2009.

[11] Jung-Han Kimn. A convergence theory for an overlapping Schwarz algorithm using discontinuous iterates. Numer. Math., 100(1):117-139, 2005.

[12] P.-L. Lions. On the Schwarz alternating method. I. In First International Symposium on Domain Decomposition Methods for Partial Differential Equations (Paris, 1987), pages 1-42. SIAM, Philadelphia, PA, 1988.

[13] P.-L. Lions. On the Schwarz alternating method. II. Stochastic interpretation and order properties. In Domain decomposition methods (Los Angeles, CA, 1988), pages 47-70. SIAM, Philadelphia, PA, 1989.

[14] P.-L. Lions. On the Schwarz alternating method. III. A variant for nonoverlapping subdomains. In Third International Symposium on Domain Decomposition Methods for Partial Differential Equations (Houston, TX, 1989), pages 202-223. SIAM, Philadelphia, PA, 1990.

[15] Sébastien Loisel and Daniel B. Szyld. On the geometric convergence of optimized Schwarz methods with applications to elliptic problems. Numer. Math., 114(4):697-728, 2010.

[16] S. Mizohata. The theory of partial differential equations. Cambridge University Press, New York, 1973. Translated from the Japanese by Katsumi Miyahara.

[17] E. Zeidler. Nonlinear functional analysis and its applications. II/B. Springer-Verlag, New York, 1990. Nonlinear monotone operators, Translated from the German by the author and Leo F. Boron. 